\title{
870nm Bragg grating in single mode TOPAS microstructured polymer optical fibre
}

\author{
Wu Yuan ${ }^{1 *}$, David J. Webb ${ }^{2}$, Kyriacos Kalli $^{3}$, Kristian Nielsen $^{1}$, Alessio Stefani ${ }^{1}$, \\ Henrik K. Rasmussen ${ }^{4}$, Ole Bang ${ }^{1}$ \\ 1 DTU Fotonik, Dept. of Photonics Engineering, Technical University of Denmark, \\ DK-2800 Kgs. Lyngby, Denmark \\ *wyuan@,fotonik.dtu.dk \\ 2 Photonic Research Group, Aston University, Aston Triangle, Birmingham, B4 7ET, UK \\ 3 Nanophotonics Research Laboratory, Cyprus University of Technology, Cyprus \\ 4 Dept. of Mechanical Engineering, Technical University of Denmark, \\ DK-2800 Kgs. Lyngby, Denmark
}

\begin{abstract}
We report the fabrication and characterization of a fiber Bragg grating (FBG) with $870 \mathrm{~nm}$ resonance wavelength in a single-mode TOPAS microstructured polymer optical fiber (mPOF). The grating has been UV-written with the phasemask technique using a $325 \mathrm{~nm} \mathrm{HeCd}$ laser. The static tensile strain sensitivity has been measured as $0.64 \mathrm{pm} / \mu$ strain, and the temperature sensitivity was $-60 \mathrm{pm} /{ }^{\circ} \mathrm{C}$. This is the first $870 \mathrm{~nm}$ FBG and the first demonstration of a negative temperature response for the TOPAS FBG, for which earlier results have indicated a positive temperature response. The relatively low material loss of the fiber at this wavelength compared to that at longer wavelengths will considerably enhance the potential utility of the TOPAS FBG.
\end{abstract}

Keywords: fiber Bragg grating, TOPAS, microstructured polymer optical fiber

\section{INTRODUCTION}

Polymer optical FBGs have shown great potential for sensor applications to sense for example temperature and strain with higher sensitivity and wider tunability than its silica counterpart [1]. Those advantages are due to the lower Young's modulus and higher thermo-optic coefficient of POFs [2]. In addition, polymers are clinically acceptable, which along with the flexible, non-brittle nature of the fibers makes these gratings an important candidate for in-vivo biosensing applications [3].

Gratings have been reported in both step index (SI) [4] and microstructured polymer optical fibres (mPOFs) $[5,6]$. Microstructured optical fibres have a pattern of air holes that extend for the full length of the fibre and the optical properties can be designed by adjusting the relative position, size and shape of the air holes. Importantly, the holes can be used to hold a gas or a biological sample, which can be studied by evanescent-wave sensing.

The majority of polymer optical fibre (POF) to date is based on polymethyl methacrylate (PMMA). Monomer residues inside PMMA, and its aptitude for water absorption often make the drawing process with commercially available PMMA rods problematic, and handicap PMMA based FBG strain sensors with a significant cross-sensitivity to humidity. These problems can be reduced by using other polymer materials, such as TOPAS ${ }^{\circledR}$ cyclic olefin copolymers (TOPAS COCs). TOPAS COCs have no monomers and their moisture absorption is a hundred times lower than PMMA. Furthermore, although TOPAS is chemically inert and bio-molecules do not readily bind to its surface, treatment with antraquinon and subsequent UV activation allows sensing molecules to be deposited in well defined spatial locations [3]. When combined with grating technology this provides considerable potential for label-free bio-sensing [7].

Despite promises not many commercial applications have been realized yet due to the high material loss of both PMMA and TOPAS mPOFs in the 1300-1500nm spectral region. A considerable decrease in the material loss from about 100 $\mathrm{dB} / \mathrm{m}$ to $1 \mathrm{~dB} / \mathrm{m}$ can be achieved by working at a lower wavelengths [8]. In this paper we report the fabrication and characterization of a FBG with $870 \mathrm{~nm}$ resonance wavelength in a single mode TOPAS mPOF. The phase-mask

21st International Conference on Optical Fiber Sensors, edited by Wojtek J. Bock, Jacques Albert, Xiaoyi Bao, Proc. of SPIE Vol. 7753, 77538X - (c) 2011 SPIE · CCC code: 0277-786X/11/\$18 · doi: 10.1117/12.884986 
technique has been used to write the grating with a $325 \mathrm{~nm}$ laser. The static tensile strain sensitivity has been measured to $0.64 \mathrm{pm} / \mu$ strain and the temperature sensitivity has been measured to $-60 \mathrm{pm} /{ }^{\circ} \mathrm{C}$. This is the smallest Bragg wavelength reported to date in a TOPAS FBG and, very importantly, it is the first confirmation of a negative temperature response for a TOPAS FBG. The relatively low material loss of the fiber at this wavelength compared to that at longer wavelengths will considerably enhance the potential utility of the TOPAS FBG, just as for the PMMA, in which an FBG was recently written at $827 \mathrm{~nm}$ [6]. Furthermore, it is also convenient to work at this particular wavelength since the CMOS technology potentially can provide interrogation systems that are even cheaper than those at $1550 \mathrm{~nm}$.

\section{EXPERIMENTS}

\subsection{TOPAS mPOFs and FBG writing}

The TOPAS mPOF used in our experiment was fabricated with low tension in order to decrease the birefringence along the fiber axis. The resulting mPOF has a diameter of $240 \mu \mathrm{m}$ and a solid core surrounded by two rings of air holes arranged in a triangular lattice. The air-hole diameter is in average $2 \pm 0.2 \mu \mathrm{m}$ and the inter-hole pitch is in average $6 \pm 0.2$ $\mu \mathrm{m}$, respectively, as shown in Fig. 1. The mPOF is endlessly single-mode with a hole diameter to pitch ratio of $\mathrm{d} / \Lambda \approx 0.33$, which is close to the theoretical limit of 0.406 for endlessly single-mode behavior of a silica Photonic Crystal Fiber (PCF) with a finite number of air-hole rings [9].

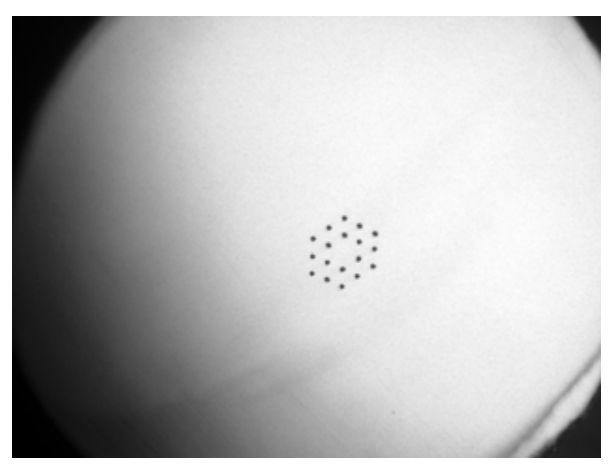

Figure 1. The microscope image of the end facet of our TOPAS mPOF.

The gratings were inscribed using a $30 \mathrm{~mW} \mathrm{CW} \mathrm{HeCd} \mathrm{laser} \mathrm{operating} \mathrm{at} 325 \mathrm{~nm}$ (IK5751I-G, Kimmon). The fiber was supported by v-grooves on both sides with a gap in between to avoid reflection, and it was appropriately stretched to ensure that the fiber did not sag. A circular gaussian laser beam was expanded from diameter $1.2 \mathrm{~mm}$ to $1.2 \mathrm{~cm}$ in one direction along the fiber by a cylindrical lens. The laser beam was then focused vertically downwards into the fiber core using another cylindrical lens to expose the fiber through a phasemask customized for $325 \mathrm{~nm}$ writing with a uniform period of $572.4 \mathrm{~nm}$ (Ibsen Photonics), designed for $850 \mathrm{~nm}$ grating inscription in PMMA. A grating length of $10 \mathrm{~mm}$ was defined by an aperture underneath the focus lens to control the beam width. The laser irradiance at the fiber was about $5 \mathrm{Wcm}^{-2}$. The resulting grating wavelength was around $870 \mathrm{~nm}$, with the longer resonance wavelength being due to the higher refractive index of TOPAS at close to $800 \mathrm{~nm}$. The phase-mask was originally chosen for use with PMMA.

The growth of the $10 \mathrm{~mm}$ gratings was monitored in reflection during the inscription using an $850 \mathrm{~nm}$ silica fiber circulator, a SuperK Versa broadband source (NKT Photonics) and an optical spectrum analyzer (Ando AQ6317B). A standard single-mode silica fiber was butt-coupled to the mPOF using an angle cleaved end-facet and a small amount of refractive index matching gel in order to reduce Fresnel reflections, which manifested themselves as background noise. The ends of the $\mathrm{mPOF}$ were prepared using a homemade hot blade cleaver equipped with flat side blade with a temperature of $50^{\circ} \mathrm{C}$ for both the blade and fiber, which gives a high quality end facet. The typical reflection spectra of a $10 \mathrm{~mm}$ grating fabricated in the TOPAS mPOF with different exposure times are shown in Fig. 2(a). The exposure time was usually much longer in the mPOF than that needed in the step-index POF to write a grating. This is because the holes around the core of the mPOF scatter a significant part of the laser power during the writing process.

The grating reflectivity was difficult to measure precisely, because the material loss and coupling loss between mPOF and silica fiber cannot be precisely measured. A rough estimate is that the reflectivity of the grating with 338 minutes exposure is about $70 \%$ by taking into account the loss of the fiber and comparing the level of the reflected grating peak 
to the Fresnel reflection from the flat end of the glass fiber. This would give a refractive index modulation of at least $4 \times 10^{-4}$. We further observe that side-lobes appear in the reflection spectrum after 236 minutes.
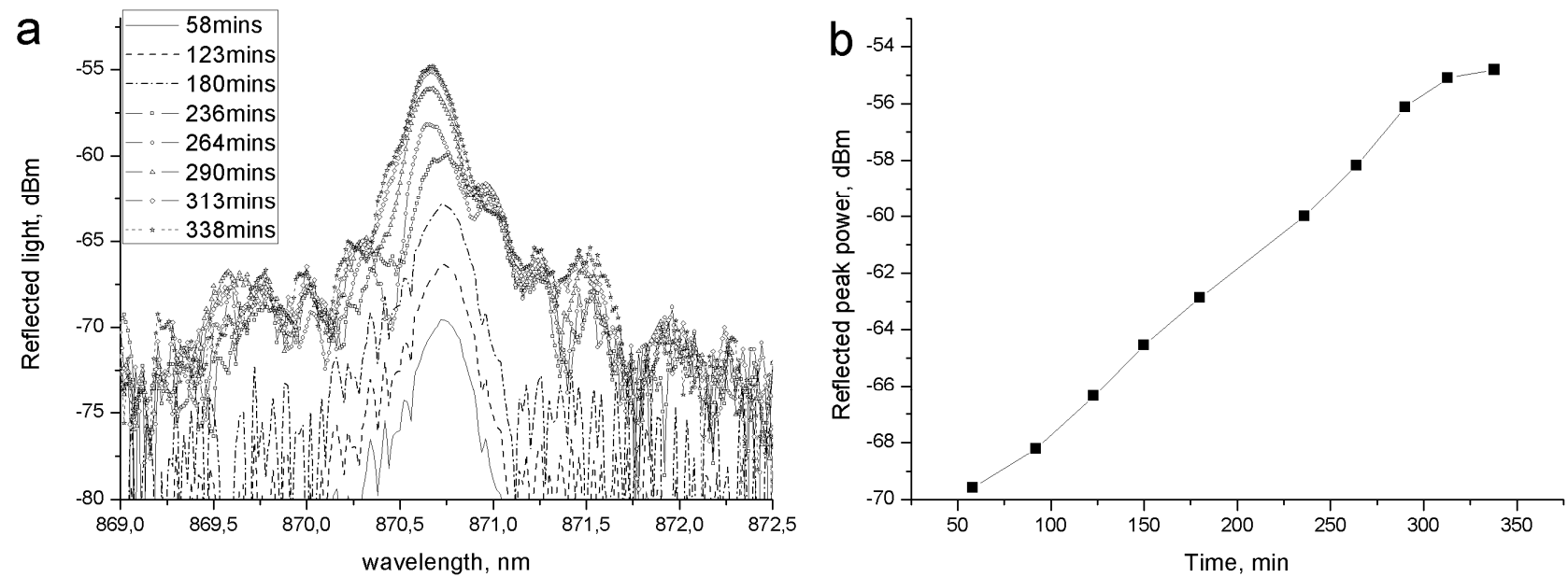

Figure 2. (a) Reflection spectra of a $10 \mathrm{~mm}$ FBG in a TOPAS mPOF at different writing times. (b) Growth dynamic of the peak intensity of the $10 \mathrm{~mm}$ FBG during writing.

The growth dynamics of the gratings, i.e. the time dependent peak intensity, is shown in Fig. 2(b). The grating began with a growth in strength accompanied by an almost constant grating bandwidth. After a certain time threshold, which was around 313 minutes, the grating strength begins to saturate.

\subsection{Strain and temperature characterization of TOPAS mPOF FBGs}

The strain tuning of the FBG was investigated by mechanical stretching. The two ends of the mPOF were glued to two micro-translation stages, with one of them fixed and used to butt-couple the mPOF to a silica single mode fiber. The other stage can move longitudinally to apply axial strain to the grating manually with a low loading speed. The axial strain values were determined by dividing the fiber longitudinal elongation by the length of fiber between the two gluing points. The longitudinal displacement accuracy of the moving translation stage is $0.01 \mathrm{~mm}$. The gratings were left to stabilize for about ten minutes each time the tensile strain was changed before reading the reflection spectrum. A single strain loading cycle experiment was carried out to study the strain tuning responses of the grating, as shown in Fig. 3(a). The fiber was gradually stretched to $2.17 \%$ strain. The grating shows a quasi-linear response of the wavelength shift over the whole strain loading range and the results indicate a strain sensitivity of $0.64 \pm 0.04 \mathrm{pm} / \mu$ strain.
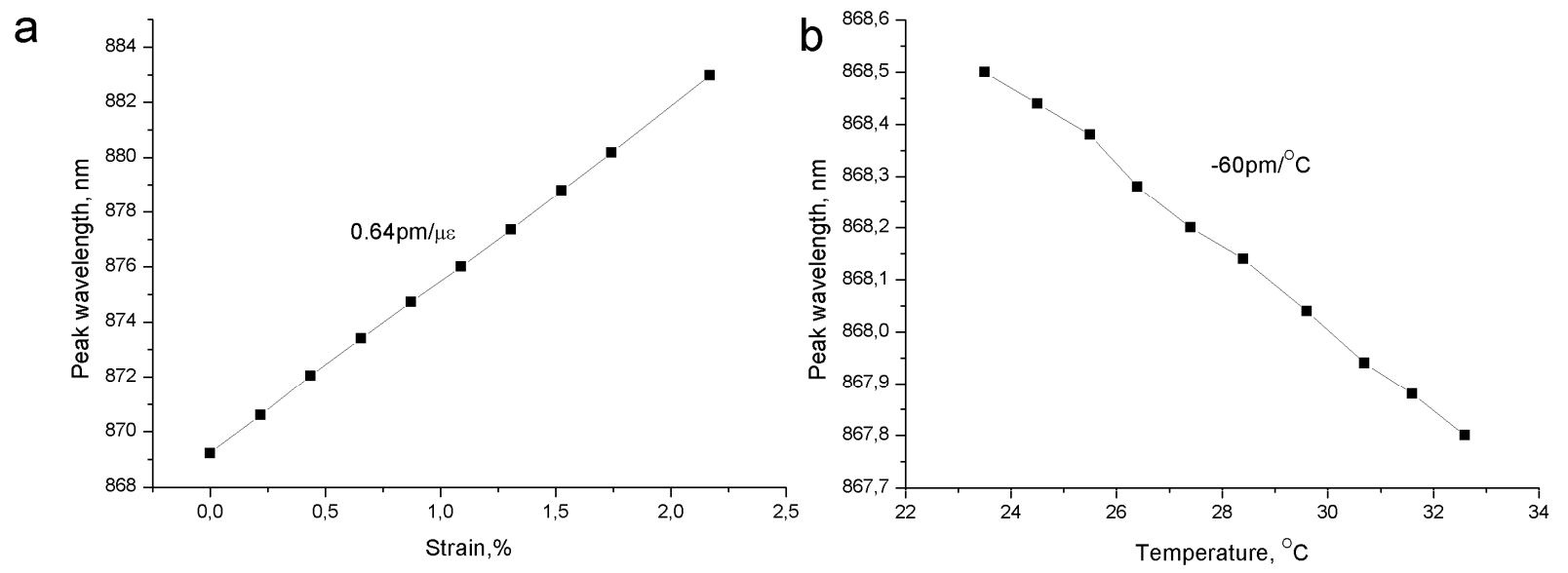

Figure 3. (a) Strain response of the $10 \mathrm{~mm}$ TOPAS mPOF FBG. (b) Temperature response of the $10 \mathrm{~mm}$ TOPAS mPOF FBG.

The temperature response of the gratings was also studied with the same monitoring setup as the one used during the grating inscription. The grating section of the polymer fiber was heated up with a resistive hot stage (MC60+TH60, 
Linkam). A thermo coupler was used to measure the temperature as close to the grating as possible with an uncertainty around $0.3^{\circ} \mathrm{C}$. One end of the $\mathrm{mPOF}$ was clamped and butt-coupled to a silica fiber circulator, and the entire length of the mPOF with grating was attached to the surface of the heater by several layers of lens papers on the top. Twenty minutes was allowed for the temperature of the grating to stabilize at each new setting before readings of the resonance wavelengths and peak intensity were taken. The grating was heated up from room temperature to $32.6^{\circ} \mathrm{C}$ stepwise in a single cycle, as shown in the Fig. 3(b). A blue shift of the resonance wavelength was indentified during the heating up process, but no obvious bandwidth change was found. A temperature sensitivity about $-60 \pm 1 \mathrm{pm} /{ }^{\circ} \mathrm{C}$ was found. This means that the negative thermo-optic coefficient overrides the positive thermo-expansion coefficient in the thermal responses of the TOPAS mPOF FBG, which is similar to the case of the PMMA FBG. This corrects earlier preliminary results that showed a strong positive response of a TOPAS FBG [7]. The FBG reported here has a clear reflection spectrum, whereas the earlier unpublished measurement showed an FBG that could only be measured in transmission [7], which is most probably the explanation of the earlier wrong result.

\section{CONCLUSIONS}

We have demonstrated the inscription of an 870nm FBG in a TOPAS mPOF for the first time. The relatively low material loss of the fiber at this wavelength compared to that at longer wavelengths, together with the convenient accessibility of the cost-effective CMOS technology at this wavelength, will considerably enhance the potential utility of the TOPAS FBG. The static tensile strain sensitivity has been measured to $0.64 \mathrm{pm} / \mu$ strain and we also found that the temperature sensitivity was negative, being measured at $-60 \mathrm{pm} /{ }^{\circ} \mathrm{C}$, which corrects earlier preliminary results showing a strong positive result [7].This is the smallest Bragg wavelength reported to date and the first confirmation of a negative temperature response for a TOPAS FBG, similar to that of a PMMA FBG.

\section{ACKNOWLEDGEMENTS}

We would like to acknowledge support from the Danish National Advanced Technology Foundation.

\section{REFERENCES}

1. H. Dobb, K. Carroll, D.J. Webb, K. Kalli, M. Komodromos, C. Themistos, G.D. Peng, A. Argyros, M.C.J. Large, M.A. van Eijkelenborg, Q. Fang, I.W. Boyd, "Grating based devices in polymer optical fibre", Proc. SPIE. 6189, 1 (2006).

2. W. Yuan, A. Stefani, M. Bache, T. Jacobsen, B. Rose, N. Herholdt-Rasmussen, F.K. Nielsen, S. Andresen, O.B. Sørensen, K.S. Hansen, O. Bang, "Improved thermal and strain performance of annealed polymer optical fiber Bragg gratings", Opt. Commun. (2010). In press

3. G. Emiliyanov, J.B. Jensen, O. Bang, P.E. Hoiby, L.H. Pedersen, E.M. Kjær, L. Lindvold, "Localized biosensing with Topas microstructured polymer optical fiber", Opt. Lett. 32, 460 (2007).

4. Z. Xiong, G.D. Peng, B. Wu, P.L. Chu, "Highly tunable Bragg gratings in single-mode polymer optical fibres", IEEE Photon. Technol. Lett. 11, 352 (1999).

5. H. Dobb, D.J. Webb, K. Kalli, A. Argyros, M.C.J. Large, M.A.van Eijkelenborg, "Continuous wave ultraviolet light induced fibre bragg gratings in few- and single-mode microstructured polymer optical fibres", Opt. Lett. 30, 3296 (2005).

6. I.P. Johnson, K. Kalli, D.J. Webb, " $827 \mathrm{~nm}$ Bragg grating sensor in multimode microstructured polymer optical fiber", Electron. Lett. 46, (2010).

7. D.J. Webb, K. Kalli, K. Carroll, C. Zhang, M. Komodromos, A. Argyros, M. Large, G. Emiliyanov, O. Bang, E. Kjaer, "Recent developments of Bragg gratings in PMMA and TOPAS polymer optical fibers", Advanced Sensor Systems and Applications III, Proc. of SPIE 6830, 683002, (2007).

8. M. C. J. Large, L. Poladian, G. Barton, M.A. van Eijkelenborg, Microstructured polymer optical fibres" (Springer, 2008).

9. B. Kuhlmey, R.C. McPhedran, C.M. de Sterke, "Modal cutoff in microstructured optical fibres", Opt. Lett. 27, 1684 (2002). 